\title{
EIGENFUNCTION EXPANSIONS ASSOCIATED WITH AN INTEGRAL OPERATOR
}

\author{
BY \\ MARVIN SHINBROT(1)
}

1. In attempts to develop a theoretical foundation to describe the behavior of subjects engaged in simple learning experiments, certain authors (see, e.g., [1]) have been led to study the eigenfunctions of the operator $K$ defined by

$$
(K r)(x)=\int_{-\infty}^{\infty} k(x-a y) r(y) d y .
$$

Here, $a$ is a real constant between zero and one, and, at least in the learning theoretical application, $k(x)$ is a probability density.

It has been conjectured that under reasonable hypotheses the eigenfunctions of $K$ are complete (in $L^{2}$, say). If this were the case, it would come as something of a surprise, for in the two extreme cases $a=0$ and $a=1$ the conjecture is certainly false. When $a=0, K$ has a one-dimensional range, and its eigenfunctions cannot be complete. When $a=1$, the only eigenfunctions are exponential polynomials [2, Theorem 146], and no nontrivial exponential polynomial can be in $L^{2}$.

It is of some interest, therefore, to attempt to derive a spectral theorem for $K$. The character of our results is of interest too. To describe them, we need some notation. Let $K_{*}$ denote the adjoint of $K$ and $\mathscr{N}\left(K_{*}^{n}\right)$ the null space of $K_{*}^{n}$. Also, define

$$
\mathscr{N}=\bigcup_{n=1}^{\infty} \mathscr{N}\left(K_{*}^{n}\right)
$$

We derive several expansion theorems. The last of them can be described as follows. Every point in a certain punctured disc about the origin is an eigenvalue of $K$. Choosing arbitrarily any point in that disc, we can find a sequence of eigenvalues having the chosen point as its only limit point and such that the corre-

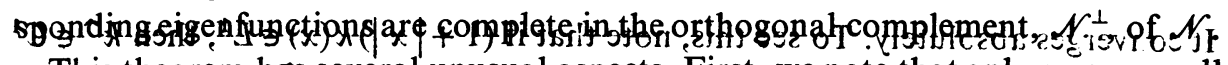

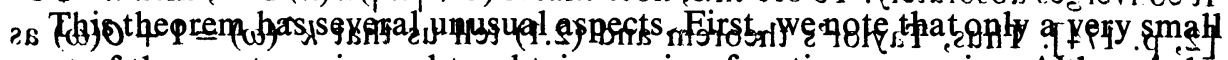

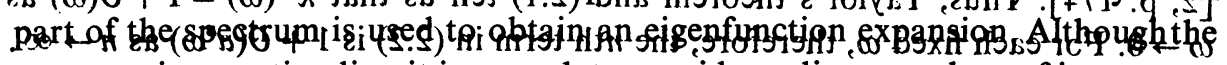

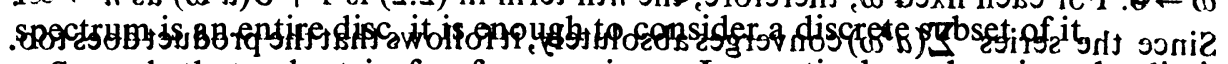

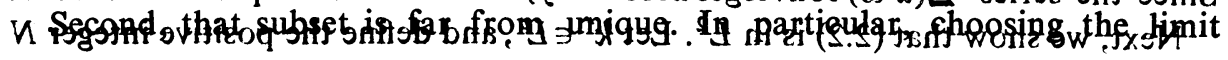

fsrlf $519225,9 W$
Received by the editors August $28,1963$.

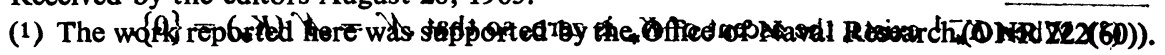


point as zero (the case of particular significance for the learning problem; see §5), we note the following interesting fact. Although $K$ is not self-adjoint (it is not even normal) and certainly is not compact, it behaves with regard to eigenfunction representation almost as if it were both: There exists a sequence of eigenfunctions whose only limit point is zero, and the corresponding eigenfunctions are complete if and only if $K_{*}^{-1}$ exists(2).

We have said that in the application, $k$ is a probability density. We shall not assume this here, since that much will not be needed. However, none of the assumptions that we do make is inconsistent with $k$ being a density. To avoid having to repeat our hypotheses in the statement of every theorem, we list them here and state once and for all that we assume them to be valid. Let $k^{\wedge}$ denote the Fourier transform of $k$. The assumptions we make, then, are:

(i) $0<a<1$,

(ii) $(1+|x|) k(x) \in L^{1}$,

(iii) $\left|k^{\wedge}(\omega)\right| \leqq\left|k^{\wedge}(0)\right|$,

(iv) $k^{\wedge} \in L^{p}$ for some finite $p>0$.

Also, to avoid trivial situations, we assume throughout that $k$ is not almost everywhere zero.

Finally, a bit more notation. The symbol $C^{n}$ will be used to denote the set of all functions with $n$ derivatives, continuous on the whole real line and approaching zero at infinity. A function will be said to be in $C_{0}^{n}$ if it is in $C^{n}$ and has compact support. As in (iii) and (iv), the Fourier transform of a function will be denoted by placing the symbol ${ }^{\wedge}$ after it.

2. Our first goal will be to prove

THEOREM 1. $k^{\wedge}(0)$ is an eigenvalue of $K$ of infinite multiplicity.

In view of (iii), $k^{\wedge}(0)$ cannot be zero. Since a multiplicative constant may be absorbed into $\lambda$ in the equation $\lambda r=K r$, we are free to assume that

$$
k^{\wedge}(0)=1 \text {. }
$$

To prove the theorem, consider the infinite product

$$
\prod_{n=0}^{\infty} k^{\wedge}\left(a^{n} \omega\right)=\hat{r_{0}}(\omega) \text {. }
$$

It converges absolutely. To see this, note that if $(1+|x|) k(x) \in L^{1}$, then $k^{\wedge} \in C^{1}$ [2, p. 174]. Thus, Taylor's theorem and (2.1) tell us that $k^{\wedge}(\omega)=1+O(\omega)$ as $\omega \rightarrow 0$. For each fixed $\omega$, therefore, the $n$th term in (2.2) is $1+O\left(a^{n} \omega\right)$ as $n \rightarrow \infty$. Since the series $\Sigma\left(a^{n} \omega\right)$ converges absolutely, it follows that the product does too.

Next, we show that (2.2) is in $L^{2}$. Let $k^{\wedge} \in L^{p}$, and define the positive integer $N$ by the inequalities $2(N-1)<p \leqq 2 N$. We assert that

(2) When $K_{*}^{-1}$ exists, the ascent of $K_{*}$ is zero, so that $\mathscr{N}=\mathscr{N}\left(K_{*}\right)=\{0\}$. 


$$
P_{N} \equiv\left[\prod_{n=0}^{N-1} k^{\wedge}\left(a^{n} \omega\right)\right]^{p / 2 N}
$$

is in $L^{2}$. This is obvious for $N=1$. Suppose $P_{N-1} \in L^{2}$. For any $q$, denote the norm in $L^{q}$ by \|\|$_{q}$. Using the Hölder inequality in the second step, we find that

$$
\begin{aligned}
\left\|P_{N}\right\|_{2}^{2} & =\left\|\prod_{n=0}^{N-1}\left|k^{\wedge}\left(a^{n} \omega\right)\right|^{p / N}\right\|_{1} \\
& \leqq\left\|\prod_{n=0}^{N-2}\left|k^{\wedge}\left(a^{n} \omega\right)\right|^{p / N}\right\|_{N /(N-1)} \cdot\left\|\left[k^{\wedge}\left(a^{N-1} \omega\right)\right]^{p / N}\right\|_{N} \\
& =\left\|P_{N-1}\right\|_{2}^{2-2 / N} \cdot\left\|k^{\wedge}\left(a^{N-1} \omega\right)\right\|_{p}^{p^{\prime N}} \\
& <\infty
\end{aligned}
$$

by the induction hypothesis and the assumption that $k^{\wedge} \in L^{p}$. Thus, $P_{N} \in L^{2}$.

By (iii) and (2.1), no term in the product

$$
\left[\prod_{n=0}^{N-1} k^{\wedge}\left(a^{n} \omega\right)\right]^{1-(p / 2 N)}\left[\prod_{n=N}^{\infty} k^{\wedge}\left(a^{n} \omega\right)\right]
$$

exceeds unity. Consequently,(2.4) is bounded. (2.2) can then be written as a product of two factors, one of which, (2.3), is in $L^{2}$, while the other, (2.4), is bounded. Therefore, (2.2) in is $L^{2}$. We can conclude that (2.2) is the transform of a function $r_{0}(x) \in L^{2}$.

This function is an eigenfunction of $K$ corresponding to the eigenvalue unity. In fact,

$$
\begin{aligned}
\hat{r_{0}}(\omega) & =\prod_{n=0}^{\infty} k^{\wedge}\left(a^{n} \omega\right) \\
& =k^{\wedge}(\omega) \prod_{n=1}^{\infty} \hat{k}\left(a^{n} \omega\right) \\
& =k^{\wedge}(\omega) \hat{r_{0}}(a \omega) .
\end{aligned}
$$

Since $k \in L^{1}$ and $r_{0} \in L^{2}$, this is equivalent to the equation $r_{0}=K r_{0}$ [2, Theorem 65]. It remains to prove the eigenvalue has infinite multiplicity. Let $s(\omega)$ be any measurable, bounded, periodic function with period $\log (1 / a)$. Define $r_{0}^{\hat{a}}$ as before, by (2.2), and let

$$
\hat{r^{\wedge}}(\omega)=\hat{r_{0}}(\omega) s(\log |\omega|),
$$

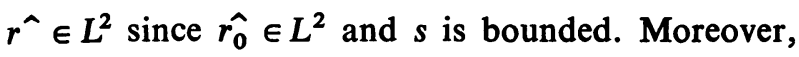




$$
\begin{aligned}
r^{\wedge}(\omega) & =\hat{r_{0}}(\omega) s(\log |\omega|) \\
& =k^{\wedge}(\omega) r_{0}^{\hat{0}}(a \omega) s(\log |\omega|) \\
& =k^{\wedge}(\omega) r_{0}(a \omega) s(\log |\omega|-\log 1 / a) \\
& =k^{\wedge}(\omega) r^{\wedge}(a \omega) .
\end{aligned}
$$

Thus, $r$, the inverse transform of $\boldsymbol{r}^{\wedge}$, is an eigenfunction of $K$, with eigenvalue one. This proves the infinite multiplicity of the eigenvalue.

3. Perhaps it is worthwhile to point out that all of the eigenfunctions we have so far discussed are not only in $L^{2}$ but also in $C^{0}$ (and so in $L^{q}$ for all $q \geqq 2$ ). To see this, we note that a similar argument to the one used before shows that $\hat{r_{0}} \in L^{1}$. Since $s$ is bounded, $\hat{r^{\wedge}}$, given by (2.5), is also in $L^{1}$, so that $r \in C^{0}$.

It is not true, however, that all eigenfunctions with eigenvalue $k^{\wedge}(0)$ are in $C^{0}$ for the assumption that $s$ is bounded was more than was needed. In fact, if $s$ is any measurable function with period $\log (1 / a)$ such that $(2.5) \in L^{2}$, the corresponding function $r$ must be an eigenfunction.

4. If, as we have assumed, $k \in L^{1}$, then $k^{\wedge} \in C^{0}$ and, in particular, $k^{\wedge}(\omega) \rightarrow 0$ as $\omega \rightarrow \infty$. Thus, the function $r_{0}$ of $\S 2$ is the product of infinitely many functions, each of which approaches zero. It may then be expected that, unless $k^{\wedge}$ goes to zero very slowly indeed (which can, however, happen), $\hat{r_{0}}$ will go to zero rather fast. In this section, which will be concerned with eigenvalues other than $k^{\wedge}(0)$, it will be seen that the spectrum of $K$ depends on how fast $r_{0}$ approaches zero.

Since $\hat{r_{0}}$ is known explicitly in terms of $\hat{k}$, we shall have no compunctions about making hypotheses that refer directly to $r_{0}$ rather than to $\hat{k}$. However, the following fact, which is a trivial consequence of the definition of $\boldsymbol{r}_{\mathbf{0}}$, should, perhaps, be noted: If there exists an $\alpha>0$ such that $|\omega|^{\alpha} k^{\wedge}(\omega) \rightarrow 0$ as $|\omega| \rightarrow \infty$, then $|\omega|^{\beta} \cdot \hat{r_{0}}(\omega) \rightarrow 0$ as $|\omega| \rightarrow \infty$ for all $\beta>0$.

To find the other eigenvalues of $K$, we shall use the easily proved

THEOREM 2. Let $k \in L^{1}$. Let $\lambda$ be an eigenvalue and $r$ the corresponding eigenfunction of $K$. If the integral of $r$ exists and does not vanish, then $\lambda=k^{\wedge}(0)$.

The proof takes less than a line: $\lambda r^{\wedge}(0)=k^{\wedge}(0) r^{\wedge}(0)$.

If $r^{\wedge}(0) \neq 0$, the corresponding eigenvalue is $k^{\wedge}(0)$. Since we wish to discuss other eigenvalues, we must assume that $r^{\wedge}(0)=0$. Accordingly, let us write

$$
\boldsymbol{r}^{\wedge}(\omega)=|\omega|^{\alpha} s(\omega),
$$

where $\operatorname{Re} \alpha>0$. If $r$ is an eigenfunction and $\lambda$ the corresponding eigenvalue, the equation $\lambda r=K r$ becomes, in its transformed version,

$$
\lambda a^{-\alpha} s(\omega)=k^{\wedge}(\omega) s(a \omega) .
$$


Solutions are obtained by taking $\lambda=a^{\alpha} k^{\wedge}(0)$ and $s$ as any of the eigenfunctions with eigenvalue $k^{\wedge}(0)$ found in $\$ 2$. Thus, we have proved part of

THeorem 3. Let $r_{0}^{\hat{0}}(\omega)$ be given by (2.2) and suppose that $|\omega|^{\alpha} r_{0}^{\hat{0}}(\omega) \in L^{2}$. Then $\lambda=a^{\alpha} k^{\wedge}(0)$ is an eigenvalue, of infinite multiplicity, of $K$. A corresponding eigenfunction is just the function whose transform is $|\omega|^{\alpha} r_{\hat{0}}(\omega)$. If $r_{0}(\omega)$ goes to zero faster than any power of $\omega$, the spectrum of $K$ is the disc of radius $\left[\left|k^{\wedge}(0)\right| / \sqrt{ } a\right]$ centered at the origin. Every point inside the punctured disc is an eigenvalue of infinite multiplicity.

We have seen that the functions

$$
r^{\wedge}(\omega)=|\omega|^{\alpha} r_{0}^{\hat{(}}(\omega)
$$

formally satisfy the eigenfunction equation

$$
\left[k^{\wedge}(0) a^{\alpha}\right] r^{\wedge}(\omega)=k^{\wedge}(\omega) r^{\wedge}(a \omega),
$$

no matter what $\alpha$ may be. Thus, $\hat{r}$ is the transform of an eigenfunction whenever it is in $L^{2}$. This is clearly the case when $-1 / 2<\operatorname{Re} \alpha \leqq 0$. If $r_{0}$ goes to zero fast enough, it is also true when $\operatorname{Re} \alpha>0$. Since the function $a^{\alpha}$ maps the half-plane $\operatorname{Re} \alpha>-1 / 2$ onto the disc of radius $1 / \sqrt{ } a$, we see that the disc of radius $\left|k^{\wedge}(0)\right| / \sqrt{ } a$ is contained in the spectrum.

On the other hand, the spectrum can contain no more than this disc, for $\|K\| \leqq\left|k^{\wedge}(0)\right| / \sqrt{ } a$. In fact, whenever $r \in L^{2}$,

$$
\begin{aligned}
\|K r\|_{2}^{2} & =\left\|(K r)^{\wedge}\right\|_{2}^{2} \\
& =\left\|k^{\wedge}(\omega) r^{\wedge}(a \omega)\right\|_{2}^{2} \\
& \leqq\left|k^{\wedge}(0)\right|^{2}\left\|r^{\wedge}(a \omega)\right\|_{2}^{2} \\
& =\frac{1}{a}\left|k^{\wedge}(0)\right|^{2}\left\|r^{\wedge}(\omega)\right\|_{2}^{2} .
\end{aligned}
$$

5. As we have seen, $K$ has many eigenfunctions. Among them, $r_{0}$ plays a special role. Our purpose in this section is to illuminate that role a little further. This will have pertinence in $\$ 6$ when we discuss an eigenfunction expansion.

Consider the situation when the kernel $k$ has compact support. (This is the case in the learning theoretical application discussed in [1].) From one point of view, this assumption cannot make any difference, for it is not incompatible with our earlier hypotheses. From another, however, things are vastly changed and simplified.

The reason is this. Let the support of $K$ be contained in an interval $[-\sigma, \sigma]$. Let $H_{\tau}$ denote the subspace of $L^{2}(-\infty, \infty)$ consisting of functions with support in $[-\tau, \tau]$. Then, as we shall presently prove, there exists a $\tau$ such that $H_{\tau}$ is an invariant subspace of $K$. On $H_{\tau}$, moreover, 


$$
(K r)(x)=\int_{-\tau}^{\tau} k(x-a y) r(y) d y \quad\left(r \in H_{\tau}\right) .
$$

As an operator on $H_{\tau}$, therefore, $K$ is compact. Consequently, its spectrum cannot be nearly as large as indicated in Theorem 3 .

Since in the application it is only the functions in $H_{\tau}$ that have significance (cf. [1]), it is worthwhile to study $K$ on that space. We shall prove that on $H_{\tau}, k^{\wedge}(0)$ is still an eigenvalue of $K$. However, in contrast to the situation on $L^{2}, k^{\wedge}(0)$ is a simple eigenvalue with eigenfunction $r_{0}$. Moreover, we shall also prove that all the eigenfunctions of $K$ on $H_{\tau}$ are related to $r_{0}$ in a very simple way.

Before proceeding with this program, we must show that $K$ maps $H_{\tau}$ into itself. We state this fact as

Lemma 1. Suppose that $k$ is zero outside an interval $[-\sigma, \sigma]$. If

$$
\tau=\frac{\sigma}{1-a}
$$

then $H_{\tau}$ is an invariant subspace of $K$.

Since the support of $k$ is in $[-\sigma, \sigma], k^{\wedge}$ is an entire function of exponential type $\sigma$. Similarly, if $r \in H_{\tau}$ (for any $\tau$ ), $r^{\wedge}$ is an entire function of exponential type $\tau$. Therefore,

$$
(K r)^{\wedge}=k^{\wedge}(\omega) \wedge(a \omega)
$$

is also entire, of type $\sigma+a \tau$. Taking $\tau$ to be defined by (5.1), we find the type of $(K r)^{\wedge}$ to be just $\tau$ again. If $k \in L^{1}$, the Paley-Wiener theorem [3] can be invoked to assert that $K r \in H_{\tau}$, as claimed.

With the machinery developed in the preceding sections, it is easy to prove the following result.

THEOREM 4. Let $k$ satisfy the conditions of Lemma 1. Then, $k^{\wedge}(0)$ is an eigenvalue of $K$. Exactly one of the corresponding eigenfunctions is in $H_{\tau}$ (where $\tau$ is related to $\sigma$ by (5.1)). Thus, as an operator on $H_{\tau}, K$ has $k^{\wedge}(0)$ as a simple eigenvalue. The corresponding eigenfunction is $r_{0}$.

The first conclusion is just Theorem 1. To prove the rest, define $r_{0}$ as before, by (2.2), and consider the functions

$$
\hat{\rho_{N}}(\omega)=\prod_{n=0}^{N} k^{\wedge}\left(a^{n} \omega\right) .
$$

Since $k^{\wedge}$ is entire, so is $\hat{\rho_{N}}$, and $\rho_{\hat{N}}$ is of exponential type

$$
\sum_{n=0}^{N} a^{n} \sigma=\frac{1-a^{N+1}}{1-a} \sigma .
$$


It follows that $r_{0} \in H_{\tau}$.

To prove $r_{0}$ to be the only such eigenfunction, let $r$ be another. Then, $r$ has compact support, and $r^{\wedge}$ is entire. In particular, $r^{\wedge}$ is continuous. Since, by hypothesis (see $(2.1)) \hat{r_{0}}(0) \neq 0$, we may write

$$
\hat{r^{\prime}}(\omega)=\hat{r_{0}}(\omega) s(\omega) \text {, }
$$

where $s(\omega)$ is continuous in a neighborhood of $\omega=0$.

Also, $s(\omega)$ satisfies the equation

$$
s(\omega)=s(a \omega)
$$

so that

$$
s(\omega)=s\left(a^{n} \omega\right)
$$

for all $n$. The continuity property of $s$ can now be seen to entail $s(\omega)=s(0)$, so that $\boldsymbol{r}^{\wedge}$ is a constant multiple of $\hat{r_{0}}$, as required.

As we saw in $\S 3, r_{0} \in C^{0}$. Actually, when $k$ has compact support, $r_{0} \in C_{0}^{0}$, so that $r_{0}$ is not only continuous in $[-\tau, \tau]$ and zero outside this interval, but goes continuously to zero at the ends. Under the conditions of Theorem 3 , however, much more than this is true. Then, in fact, $|\omega|^{n} r_{0} \in L^{1}$ for any $n$, so that $r_{0} \in C_{0}^{\infty}$. This is crucial for

THEOREM 5. Let $k$ satisfy the conditions of Lemma 1, and let $\boldsymbol{r}_{\mathbf{0}}$ approach zerofaster than any power. Then, for any non-negative integer $n, \lambda_{n}=a^{n} k^{\wedge}(0)$ is an eigenvalue of $K$ with a corresponding eigenfunction in $H_{\tau}$. For each $n$ there is only one such eigenfunction, and it is the derivative $r_{0}^{(n)}$.

To avoid carrying the constant factor $k^{\wedge}(0)$ in the computations, normalize $k$ as before, by (2.1). Define $r_{n}=r_{0}^{(n)}$, and consider

$$
\begin{aligned}
a^{n} r_{n}^{\hat{n}}(\omega) & =a^{n}(-i \omega)^{n} \hat{r_{0}}(\omega) \\
& =a^{n}(-i \omega)^{n} \hat{k}^{\wedge}(\omega) \hat{r_{0}}(a \omega) \\
& =k^{\wedge}(\omega) \hat{r_{n}}(a \omega) .
\end{aligned}
$$

Thus, $a^{n}$ is an eigenvalue with corresponding eigenfunction $r_{n} . r_{n} \in H_{\tau}$, since $r_{0} \in C_{0}^{\infty} \cap H_{\tau}$.

Again, let $\rho_{n}$ be another such eigenfunction. According to Theorem 2, $\hat{\rho_{n}} \hat{n}(0)=0$. Since $\hat{\rho_{n}}$ is entire, we may set $\hat{\rho_{n}} \hat{(\omega)}=\omega^{\mu_{n}} \sigma_{n}(\omega)$ for some integer $\mu_{n}$ and some entire function $\sigma_{n}$ that does not vanish at $\omega=0$. The eigenfunction equation gives

$$
\sigma_{n}(\omega)=a^{\mu_{n}-n} \hat{k}(\omega) \sigma_{n}(a \omega),
$$

so that 


$$
\sigma_{n}(\omega)=\left[a^{N\left(\mu_{n}-n\right)} \prod_{m=0}^{N-1} k^{\wedge}\left(a^{m} \omega\right)\right] \sigma_{n}\left(a^{N} \omega\right) .
$$

If $\mu_{n}-n>0$, it follows that $\sigma_{n}$ is identically zero, since $a<1$. If $\mu_{n}-n<0$, $\sigma_{n}$ must be infinite almost everywhere. Thus, $\mu_{n}=n$. It now follows from (5.1) (with $\mu_{n}=n$ ) and Theorem 5 that $\sigma_{n}$ is a constant multiple of $r_{0}$. Consequently, apart from a constant factor, $\rho_{n}$ is $r_{n}$, and this completes the proof.

6. In this section, we derive a sort of spectral theorem for $K$. Although the spectrum of $K$ is an entire disc, an expansion theorem will be found involving only the eigenvalues $a^{n} k^{\wedge}(0)$ and the corresponding eigenfunctions $r_{n}=r_{0}^{(n)}$. As we pointed out in $\S 5$, these are the only eigenfunctions of interest in the application.

First, we need

LEMMA 2. Let $e^{\delta|x|} f(x) \in L^{1}$ for some $\delta>0$. Then, the equations

$$
\int_{-\infty}^{\infty} x^{n} f(x) d x=0, \quad n=0,1, \cdots
$$

imply that $f$ is zero almost everywhere.

The following proof is a modification of one due to Hardy [4]. Consider

$$
\begin{aligned}
\int_{-\infty}^{\infty} f(x) \cos \omega x d x & =\int_{-\infty}^{\infty} f(x) \sum \frac{(-1)^{n} \omega^{2 n} x^{2 n}}{(2 n) !} d x \\
& =\Sigma \frac{(-1)^{n} \omega^{2 n}}{(2 n) !} \int_{-\infty}^{\infty} x^{2 n} f(x) d x \\
& =0
\end{aligned}
$$

by (6.1), the interchange being justified for $|\omega|<\delta$ by the convergence of

$$
\int_{-\infty}^{\infty}|f(x)| \Sigma \frac{|\omega x|^{2 n}}{(2 n) !} d x=\int_{-\infty}^{\infty} f(x) \cosh |\omega x| d x .
$$

In a similar way, we can show that

$$
\int_{-\infty}^{\infty} f(x) \sin \omega x d x=0
$$

for $|\omega|<\delta$. Thus, $f^{\wedge}(\omega)=0$ for $|\omega|<\delta$. But $e^{\delta|x|} f(x) \in L^{1}$, so that $f^{\wedge}(\omega)$ is analytic in the strip $|\operatorname{Im} \omega|<\delta$. Thus, $f^{\wedge}(\omega) \equiv 0$ and the lemma follows.

The condition $e^{\delta|x|} f(x) \in L^{1}$ is rather strong and in Theorem 7 below we shall assume (essentially) that $k^{\wedge}$ satisfies it. To see that we shall not be assuming too much (or at least not much too much), let us note that the condition cannot be replaced by $e^{\delta|x|^{\alpha}} f(x) \in L^{1}$ for any $\alpha<1$. For, the function 


$$
f(x)=|x| \exp \left(-|x|^{\alpha} \cos \frac{\alpha \pi}{2}\right) \quad \sin \left(|x|^{\alpha} \sin \frac{\alpha \pi}{2}\right)
$$

clearly satisfies $e^{\delta|x|^{\alpha}} f(x) \in L^{1}$. On the other hand, it also satisfies (6.1) whenever $0<\alpha<1$, as a straightforward computation shows.

We shall also need

Lemma 3. Define the set $\mathscr{N}$ by (1.1). Then,

$$
\mathscr{N}=\left\{f \in L^{2}: f^{\wedge}(\omega) \hat{r_{0}}(\omega)=0 \text { a.e. }\right\},
$$

so that $\mathscr{N}$ is a closed linear subspace of $L^{2}$.

Consider the operator $K_{*}$. It is defined explicitly by the formula

$$
\left(K_{*} f\right)^{\wedge}=\frac{1}{a} \overline{k^{\wedge}\left(\frac{\omega}{a}\right)} f^{\wedge}\left(\frac{\omega}{a}\right),
$$

the bardenoting the complex conjugate. Thus, $f \in \mathscr{N}\left(K_{*}\right)$ if and only if $k^{\wedge}(\omega) f^{\wedge}(\omega)$ is zero almost everywhere. In a similar way, one can see that $f \in \mathscr{N}\left(K_{*}^{N}\right)$ if and only if

$$
f^{\wedge}(\omega) \prod_{n=0}^{N-1} k^{\wedge}\left(a^{n} \omega\right)
$$

is zero almost everywhere. It is now but a step to prove (6.2). That $\mathscr{N}$ is closed is then a consequence of the fact that $r_{0}$ is bounded.

Since $\mathscr{N}$ is closed, it makes sense to speak of its orthogonal complement and of the orthogonal projection on $\mathscr{N}$. We denote these objects by $\mathscr{N}^{\perp}$ and $P$, respectively.

The problem of finding a spectral resolution of $K$ is, of course, the same as that of finding projections that reduce it. As a first step in this program, we note that $P$ does this job.

THEOREM 6. $P$ reduces $K$. Moreover, if $r$ is any eigenfunction of $K$ with a nonzero eigenvalue, $\operatorname{Pr}=0$.

We have to show first that $P K=K P$. Define the set $E$ to be the set of all real zeros of $r_{0} \hat{(}(\omega)$, and let $\chi$ be the characteristic function of the complement of $E$. Then, $P$ is just multiplication by $\chi$ :

Therefore,

$$
(P f)^{\wedge}=\chi(\omega) f^{\wedge}(\omega) .
$$

$$
(K P f)^{\wedge}=k^{\wedge}(\omega) \chi(a \omega) f^{\wedge}(a \omega)
$$

while

$$
(P K f)^{\wedge}=\chi(\omega) k^{\wedge}(\omega) f^{\wedge}(\omega)
$$


If $k^{\wedge}(\omega)=0,(6.3)$ and (6.4) are obviously equal. Now, $\chi(\omega)$ is zero if and only if $\hat{r_{0}}(\omega)$ is. At any point where $k^{\wedge}(\omega) \neq 0$, however, $r_{0}(\omega)$ is zero if and only if $\hat{r_{0}}(a \omega)$ is zero, as $(2.2)$ shows. Thus, at those points where $k^{\wedge}(\omega) \neq 0, \chi(\omega)=\chi(a \omega)$ and (6.3) and (6.4) are again equal. This proves the first part of Theorem 6.

The second part tells us that none of the eigenfunctions we have so far considered can be in $\mathscr{N}$. Its proof is very simple. If $f \in \mathscr{N}$, there is an integer $n$ such that $K_{,}^{n} f=0$. Letting parentheses denote the inner product in $L^{2}$, we see that if $r$ satisfies the conditions of the theorem,

$$
\begin{aligned}
(r, f) & =\frac{1}{\lambda^{n}}\left(K^{n} r, f\right) \\
& =\frac{1}{\lambda^{n}}\left(r, K_{*}^{n} f\right) \\
& =0
\end{aligned}
$$

Thus, $r \in \mathscr{N}^{\perp}$ and $\operatorname{Pr}=0$.

We now derive

THEOREM 7. Suppose that for some $\delta>0, k^{\wedge}(\omega)=O\left(e^{-\delta|\omega|}\right)$ as $\omega \rightarrow \pm \infty$. Then, the function $r_{0}(x)$ is the restriction to the real axis of a function analytic in the strip

$$
|\operatorname{Im} x|<\frac{\delta}{1-a}
$$

All its derivatives, as well as $r_{0}$ itself, are eigenfunctions of $K$, the eigenvalue corresponding to the nth derivative being $a^{n} k^{\wedge}(0)$. Moreover, the eigenfunctions $r_{0}^{(n)}$ span the space $\mathscr{N}^{\perp}$. Finally, if the set of real zeros of $k^{\wedge}(\omega)$ has zero Lebesgue measure, $\mathscr{N}=\{0\}$, so that the sequence $\left\{r_{0}^{(n)}\right\}$ is complete.

As usual, let us normalize $k$ by (2.1). Then, by hypothesis, $\left|k^{\wedge}\right| \leqq 1$, so that

$$
\begin{aligned}
\left|\hat{r_{0}}(\omega)\right| & \leqq \prod_{n=0}^{N-1}\left|k^{\wedge}\left(a^{n} \omega\right)\right| \\
& =o\left(\exp \left[-\delta|\omega| \cdot \frac{1-a^{N}}{1-a}\right]\right) \text { as } \omega \rightarrow \pm \infty
\end{aligned}
$$

for every positive integer $N$. Let $x$ be a complex number in the strip (6.5). Choosing $N$ so large that

$$
|\operatorname{Im} x|<\delta \frac{1-a^{N}}{1-a}
$$

we see from (6.6) that the function 


$$
r_{0}(x)=\frac{1}{2 \pi} \int_{-\infty}^{\infty} e^{-i \omega x} r_{0}(\omega) d \omega
$$

is defined at $x$. It is also analytic there for, by (6.6) and (6.7), differentiation can be carried out under theintegral sign. That all the derivatives $r_{0}^{(n)}$ are eigenfunctions with corresponding eigenvalues $a^{n}$ now follows as in the proof of Theorem 5 .

To prove the completeness, we have to show that whenever $f \in \mathscr{N}^{\perp}$, the equations

$$
\int_{-\infty}^{\infty} f(x) r_{0}^{(n)}(x) d x=0, \quad n=0,1, \cdots
$$

imply that $f$ is almost everywhere zero. Let $f$ satisfy (6.8). Then, by virtue of Parseval's equation,

$$
\int_{-\infty}^{\infty} f^{\wedge}(\omega) \hat{r_{0}}(\omega) \omega^{n} d \omega=0, \quad n=0,1, \cdots .
$$

But $f^{\wedge}(\omega) \hat{r_{0}}(\omega)$ satisfies the conditions of Lemma 2 , so that

$$
f^{\wedge}(\omega) \hat{r_{0}}(\omega)=0 \text { a.e. }
$$

By Lemma 3, this means that $f \in \mathscr{N}$. If $f \in \mathscr{N}^{\perp}$ also, $f$ must be zero almost everywhere, as we wanted to prove.

The last sentence of the theorem is an immediate consequence of Lemma 3, for if $k^{\wedge}$ is zero on a set of measure zero only, the same is true of $r_{0} \hat{\text {. Thus, }}$ (6.2) gives that $f \in \mathscr{N}$ if and only if $f^{\wedge}$ (and, therefore, $f$ ) is zero almost everywhere.

7. As we have pointed out, the growth condition in Theorem 8 is very restrictive. It excludes many interesting cases, the most important occurring when $k$ has compact support. (If $\hat{k}(\omega)=O\left(e^{-\delta|\omega|}\right), k$ is the restriction to the real axis of an analytic function; it cannot, therefore, have compact support.) It is our purpose in this section to derive a theorem without use of the offending condition.

There is difficulty here. As the example following the proof of Lemma 3 shows, the eigenfunctions $r_{0}^{(n)}$ cannot be expected to be complete (even when $\mathscr{N}=\{0\}$ ) unless a strong growth condition is satisfied. The possibility of getting around this by utilizing the other eigenfunctions of $K$ will be explored in $\S 8$. Because of the discussion in $\$ 5$, however, we proceed otherwise for now. As will be seen, it is possible to derive an interesting and unusual form of expansion theorem even when $k^{\wedge}$ is not exponentially small at infinity.

Set

$$
k^{\wedge}(\omega, \delta)=e^{-\delta|\omega|} k^{\wedge}(\omega)
$$

and define the operator $K(\delta)$ by

$$
[K(\delta) r](x)=\int_{-\infty}^{\infty} k(x-a y, \delta) r(y) d y .
$$


The eigenfunctions of $K(\delta)$ depend on those of $K$ in a very simple way, as the following result shows.

THEOREM 8. If $r$ is any eigenfunction of $K$, then the function whose transform is

$$
\hat{r}^{\wedge}(\omega, \delta)=e^{-\delta|\omega| /(1-a)} \hat{r^{\wedge}}(\omega)
$$

is an eigenfunction of $K(\delta)$ with the same eigenvalue as $r$. In particular, defining

$$
\hat{r_{0}}(\omega, \delta)=e^{-\delta|\omega| /(1-a)} \hat{r_{0}}(\omega),
$$

the functions

$$
r_{0}^{(n)}(x, \delta)=\frac{\partial^{n}}{\partial x^{n}} r_{0}(x, \delta)
$$

are all eigenfunctions of $K(\delta)$ with eigenvalues $a^{n} k^{\wedge}(0)$.

In fact,

$$
\begin{aligned}
k^{\wedge}(\omega, \delta) r^{\wedge}(a \omega, \delta) & =\left[e^{-\delta|\omega|} k^{\wedge}(\omega)\right]\left[e^{-a \delta|\omega| /(1-a)} \hat{r^{\prime}}(a \omega)\right] \\
& =\lambda \hat{r^{\wedge}}(\omega, \delta)_{*}
\end{aligned}
$$

if $r$ has the eigenvalue $\lambda$. The rest of the theorem is just the first part of Theorem 7 applied to $K(\delta)$.

$k(x, \delta)$ satisfies all the conditions of Theorem 7. Also, since the zeros of $k^{\wedge}(\omega, \delta)$ and $k^{\wedge}(\omega)$ coincide,

$$
\begin{aligned}
\mathscr{N}(\delta) & =\bigcup_{n=0}^{\infty} \mathscr{N}\left(K_{*}^{n}(\delta)\right) \\
& =\mathscr{N},
\end{aligned}
$$

as Lemma 3 shows. Thus, the sequence $\left\{r_{0}^{(n)}(x, \delta)\right\}$ is complete in $\mathscr{N}^{\perp}$, a set that depends only on $K$ and not on $\delta$.

We have therefore proved

THEOREM 9. The function $r_{0}(x, \delta)$ defined by

$$
r_{0}(x, \delta)=e^{-\delta|\omega| /(1-a)} \hat{r_{0}}(\omega)
$$

is an eigenfunction of $K(\delta)$ for every $\delta>0$, as are all of its derivatives. These eigenfunctions span $\mathscr{N}^{\perp}$. If the set of real zeros of $k^{\wedge}(\omega)$ has Lebesgue measure zero, $P=0$, so that $r_{0}(x, \delta)$ and its derivatives are complete in $L^{2}$.

Because of the importance of that case, it is worthwhile mentioning that if $k$ has compact support, the set of zeros of $k^{\wedge}$ has measure zero, so that $P=0$. For then, $\hat{k}$ is entire. 
8. We have just seen that the functions $r_{0}^{(n)}$ (or at least approximations to them) are complete in $\mathscr{N}^{\perp}$. However, this result is somewhat unsatisfactory since the functions $r_{0}^{(n)}(x, \delta)$ are not eigenfunctions of $K$, but of $K(\delta)$. To obtain an expansion in terms of eigenfunctions of $K$ itself, it is necessary to abandon the restriction to the functions $r_{0}^{(n)}$. We shall do this now and shall see that Theorem 9 is a special case of the much more general result described in $\$ 1$.

We shall, in fact, prove

THEOREM 10. Let $\lambda_{\infty}$ be any point in the annulus

$$
\left|k^{\wedge}(0)\right| \leqq\left|\lambda_{\infty}\right| \leqq\left|k^{\wedge}(0)\right| / \sqrt{ } a \text {. }
$$

Then, there exists a sequence $\left\{\lambda_{n}\right\}$ of eigenvalues and a sequence $\left\{r_{n}\right\}$ of corresponding eigenfunctions of $K$ with the following properties:

I. $\lambda_{n} \rightarrow \lambda_{\infty}$ as $n \rightarrow \infty$;

II. the sequence $\left\{r_{n}\right\}$ is complete in $\mathscr{N}^{\perp}$. If, for some $\beta>-1 / 2,|\omega|^{\beta} r_{0}(\omega) \in L^{2}, \lambda_{\infty}$ may be taken to lie in

$$
\left|k^{\wedge}(0)\right| a^{\beta} \leqq\left|\lambda_{\infty}\right| \leqq\left|k^{\wedge}(0)\right| a^{-1 / 2},
$$

and if $r_{0}(\omega)$ approaches zero faster than any power, $\lambda_{\infty}$ may be any point in the disc

$$
\left|\lambda_{\infty}\right| \leqq\left|k^{\wedge}(0)\right| a^{-1 / 2} .
$$

Since $\omega^{\beta} r_{0}(\omega)$ is always in $L^{2}$ if $-1 / 2<\beta \leqq 0$ the last part of the theorem implies the first. It therefore suffices to assume $|\omega|^{\beta} r_{0}(\omega) \in L^{2}$ and $\lambda_{\infty}$ lies in (8.1).

We have seen in Theorem 3 that, with this hypothesis, the interior of (8.1) is in the point spectrum of $K$, and that if $\lambda=k^{\wedge}(0) a^{\alpha}$, an eigenfunction with this eigenvalue is just the inverse transform of $|\omega|^{\alpha} r_{\hat{0}} \hat{(}(\omega)$. It can be verified immediately that the transform of another such eigenfunction is $|\omega|^{\alpha}{ }^{\alpha} \hat{\gamma}(\omega) \operatorname{sgn} \omega$.

If Theorem 10 were false, then, there would exist a function $f \in \mathscr{N}^{\perp}$ such that

$$
\int_{0}^{\infty} f^{\wedge}(\omega) r_{0}^{\wedge}(\omega) \omega^{\alpha_{n}} d \omega
$$

is zero for any sequence $\left\{\alpha_{n}\right\}$ lying entirely in the strip $-1 / 2<\operatorname{Re} \alpha<\beta$ and whose limit, $\alpha_{\infty}$ say, satisfies

$$
\lambda_{\infty}=k^{\wedge}(0) a^{\alpha_{\infty}} .
$$

If $\lambda_{\infty} \neq 0$, it follows that the function

$$
\phi(\alpha)=\int_{0}^{\infty} f^{\wedge}(\omega) r_{0}^{\hat{\gamma}}(\omega) \omega^{\alpha} d \omega
$$


isidentically zero in a neighborhood of $\alpha_{\infty}$. But $\phi(\alpha)$ is analytic for $-1 / 2<\operatorname{Re} \alpha<\beta$; its derivative, in fact, is

$$
\phi^{\prime}(\alpha)=\int_{0}^{\infty} f^{\wedge}(\omega) \hat{r_{0}}(\omega) \omega^{\alpha} \log \omega d \omega .
$$

Consequently, $\phi(\alpha) \equiv 0$ for $-1 / 2<\operatorname{Re} \alpha<\beta$. Writing $\phi$ in the form

$$
\phi(x)=\int_{-\infty}^{\infty}\left[f^{\wedge}\left(e^{t}\right) r_{0}\left(e^{t}\right)\right] e^{\alpha t} d t,
$$

we see that it is a two-sided Laplace transform zero in a strip. This is enough $[5$, Theorem VI.6.6b] to imply that

$$
f^{\wedge}(\omega) r_{0}^{\wedge}(\omega)=0
$$

for almost all positive $\omega$. A similar proof applies when $\lambda_{\infty}=0$.

Since we can show just as easily that (8.2) holds when $\omega$ is negative, we conclude from Lemma 3 that $f \in \mathscr{N}$. By assumption, $f \in \mathscr{N}^{\perp}$, so that $f=0$ almost everywhere. This completes the proof.

\section{REFERENCES}

1. Michael V. Levine, Ph. D. Thesis, Stanford University, Stanford, Calif. To appear.

2. E. C. Titchmarsh, Introduction to the theory of Fourier integrals, 2nd ed., Clarendon Press, Oxford, 1948.

3. R. P. Boas, Jr., Entire functions, Academic Press, New York, 1954.

4. G. H. Hardy, On Stieltjes' "probleme des moments," Messenger of Math. 46 (1917), 175-182.

5. D. V. Widder, The Laplace transform, Princeton Univ. Press, Princeton, N. J., 1946.

University of California,

Berkeley, California 